Revista de Matemática: TeOría y APliCACiones 2016 23(1) : 111-142

CIMPA - UCR ISSN: 1409-2433 (PRINT), 2215-3373 (ONLINE)

\title{
SC: A NOVEL FUZZY CRITERION FOR SOLVING ENGINEERING AND CONSTRAINED OPTIMIZATION PROBLEMS
}

\author{
SC: UN NUEVO CRITERIO DIFUSO PARA \\ RESOLVER PROBLEMAS DE INGENIERÍA Y DE \\ OPTIMIZACIÓN CON RESTRICCIONES
}

\author{
Sergio G. DE-LOS-CobOS-Silva* \\ Miguel A. GutiérRez-ANDrAdE ${ }^{\dagger}$ ERIC A. RinCÓN-GARCÍA \\ PEDro LARA-VElÁZQUEZ ${ }^{\S}$ ROMAN A. MORA-GUTIÉrREZ ${ }^{\mathbb{I}}$ \\ ANTONIN S. PONSICH"
}

Received: 2 Oct 2014; Revised: 5 Oct 2015;

Accepted: 6 Oct 2015

*Universidad Autónoma Metropolitana-Iztapalapa, Departamento de Ingeniería Eléctrica, Av. San Rafael Atlixco 186, Col. Vicentina, Del. Iztapalapa, México D.F., C.P. 09340, México. EMail: cobos@xanum.uam.mx

'Same address as S. de-los-Cobos-Silva. E-Mail: gamma@xanum.uam.mx

*Universidad Autónoma Metropolitana-Azcapotzalco, Departamento de Sistemas, Av. San Pablo 180, Colonia Reynosa Tamaulipas, México D.F., C.P. 02200, México. E-Mail: rigaeral@correo.azc.uam.mx

${ }^{8}$ Same address as S. de-los-Cobos-Silva. E-Mail: plara@xanum.uam.mx

${ }^{\text {II }}$ Same address as E. Rincón-García. E-Mail: mgra@correo.azc.uam.mx

"Same address as E. Rincón-García. E-Mail: aspo@correo@correo.azc.uam.mx 


\begin{abstract}
In this paper a novel fuzzy convergence system (SC) and its fundamentals are presented. The model was implemented on a monoobjetive PSO algorithm with three phases: 1) Stabilization, 2) generation and breadthfirst search, and 3) generation and depth-first. The system SC-PSO-3P was tested with several benchmark engineering problems and with several CEC2006 problems. The computing experience and comparison with previously reported results is presented. In some cases the results reported in the literature are improved.
\end{abstract}

Keywords: particle swarm optimization (PSO); optimization.

\title{
Resumen
}

En este trabajo se presenta un novedoso sistema de convergencia (SC), sus fundamentos y la experiencia computacional. Se implementó en un algoritmo PSO monoobjetivo de tres fases: Estabilización, generación y búsqueda en amplitud, generación y búsqueda a profundidad, el cual se probó con diversos problemas benchmark tanto de ingeniería como de la serie CEC2006. La experiencia computacional y la comparación con resultados previamente reportados se presenta. En algunos casos, se mejoran los resultados de la literatura.

Palabras clave: optimización por enjambres de partículas; optimización.

Mathematics Subject Classification: 90C26, 90C29, 90C59.

\section{Introduction}

In general, an optimization problem can be defined as:

$$
\min _{x \in X} h(x)
$$

Many real world problems can be expressed as continuous optimization problems, either with or without restrictions. Due to the complexity and intractability of many of these problems different heuristic solution methods have been developed, among others: Tabu Search (TS) [9], Simulated Annealing (SA) [21], Genetic Algorithms (GA) [15], Scatter Search (SS) [10], Particle Swarm Optimization (PSO) [19], just for PSO, there are so many different versions that a treaty on its taxonomy can be found in [31]. For a review of its variants, see [16].

With respect to constrained optimization: Toscano-Pulido and Coello (2004) [33]presented a simple mechanism to handle constraints with PSO based on 


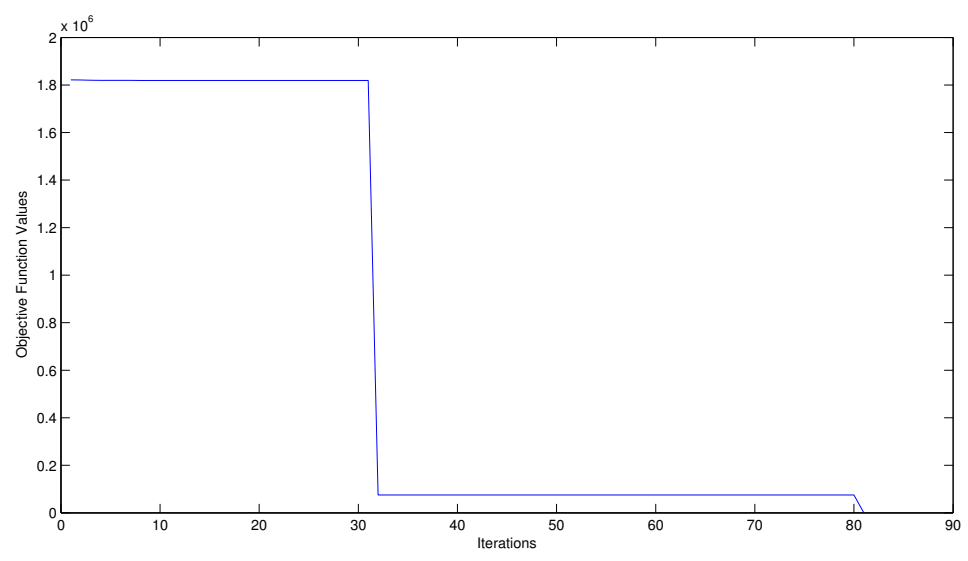

Figure 1: Convergence with SC for Griewank function dimension=120,000.

closeness of a particle to feasible region in order to select a leader, this approach also incorporates a mutation operator. The authors perform 340,000 objective function evaluations. Lu \& Chen (2008) [26] presented an algorithm called selfadaptive velocity PSO. To handle constrains, they adopted a dynamic-objective constraint-handling method. Liu et al. (2010) [25] proposed an hybrid PSO named PSO-DE, which integrates PSO with differential evolution (DE) in order to force jump out of stagnation. Mazhoud et al. (2013) [27] proposed constrainthandling mechanism consists of closeness evaluation of a particle to the feasible region, at each iteration, the constraint total violation function is introduced as a second objective, in order to solve the bi-objective optimization problem they use a basic lexicographic method, the algorithm is named CVI-PSO and the algorithms have been tested over 24 benchmark functions with 25,000 fitness function evaluations(FFE) for problems with 2 until 24 variables.

In particular, with the SC-PSO-3P model, evidence of convergence for the Grienwank function with 120,000 variables $x_{i} \in[-600,600]$ is presented (see Figure 11 using only 3 particles and reaching global optimum in less than 90 iterations on average and 40 seconds on average. The instances were solved using Matlab and running it on a Notebook with an Intel Atom N280 processor at $1.66 \mathrm{Ghz}$. Subsequently, in the section of numerical examples, an Intel Core i5-3210M processor computer at $2.5 \mathrm{GHz}$ computer was used.

This paper presents a novel approach that guarantees the solution in a single algorithm. The work is divided as follows: background of fuzzy numbers are presented in the second section; the proposed Convergence System SC, as well as its definitions and fundamentals are presented in the third section. The gen- 
eral guidelines of PSO are presented in the fourth section. Numerical examples are presented in the fifth section. Finally, conclusions and future research are presented.

\section{Fuzzy numbers}

In this section, we introduce the basic concepts of fuzzy numbers based on [6].

A fuzzy number $A=(a, b, c, d ; w)$ is defined as a fuzzy subset of the real line $\Re$ with membership function $h_{A}$ such that:

1. $h_{A}$ is continuous mapping from $\Re$ to the closed interval $[0, w]$.

2. $h_{A}=0, \forall x \in(-\infty, a]$.

3. $h_{A}$ is a strictly increasing function on $[a, b]$.

4. $h_{A}=w, \forall x \in[b, c]$, where $w$ is a constant and $0 \leq w \leq 1$.

5. $h_{A}$ is strictly decreasing on $[c, d]$.

6. $h_{A}=0, \forall x \in[d,+\infty)$,

where $0 \leq w \leq 1$, and $a, b, c, d \in \mathbb{R}$, and $a \leq b \leq c \leq d$.

If $w=1$, the generalized fuzzy number $A$ is called a normal trapezoidal fuzzy number (see Fig. 2 denoted as $A=(a, b, c, d)$. If $a=b$ and $c=d$, then $A$ is a crisp interval. If $b=c$, then $A$ is a generalized triangular fuzzy number. If $a=b=c=d$, then $A$ is a real number.

The membership function $h_{A}$ of $A$ can be expressed as:

$$
h_{A}= \begin{cases}h_{A}^{L}(x), & a \leq x \leq b, \\ w & b \leq x \leq c, \\ h_{A}^{R}(x), & c \leq x \leq d, \\ 0, & \text { otherwise }\end{cases}
$$

where: $h_{A}^{L}(x):[a, b] \longrightarrow[0, w]$ and $h_{A}^{R}(x):[c, d] \longrightarrow[0, w]$ are continuous, $h_{A}^{L}(x)$ is strictly increasing and $h_{A}^{R}(x)$ strictly decreasing. The inverse functions of $h_{A}^{L}(x)$ and $h_{A}^{R}(x)$ are denoted by $g_{A}^{L}(x)$ and $g_{A}^{R}(x)$, respectively. These functions are continuous on $[0, w]$, this means both $\int_{0}^{w} g_{A}^{L}(x)$ and $\int_{0}^{w} g_{A}^{R}(x)$ exist [23].

Let be two trapezoidal fuzzy numbers. $\tilde{B}_{1}=\left(a_{1}, b_{1}, c_{1}, d_{1} ; w\right)$ and $\tilde{B}_{2}=$ $\left(a_{2}, b_{2}, c_{2}, d_{2} ; w\right)$ and $c \in \mathbb{R}$, then:

1. $c \tilde{B}_{1}=\left(c a_{1}, c b_{1}, c c_{1}, c d_{1} ; w\right)$.

2. $\tilde{B}_{1}+\tilde{B}_{2}=\left(a_{1}+a_{2}, b_{1}+b_{2}, c_{1}+c_{2}, d_{1}+d_{2} ; w\right)$. 


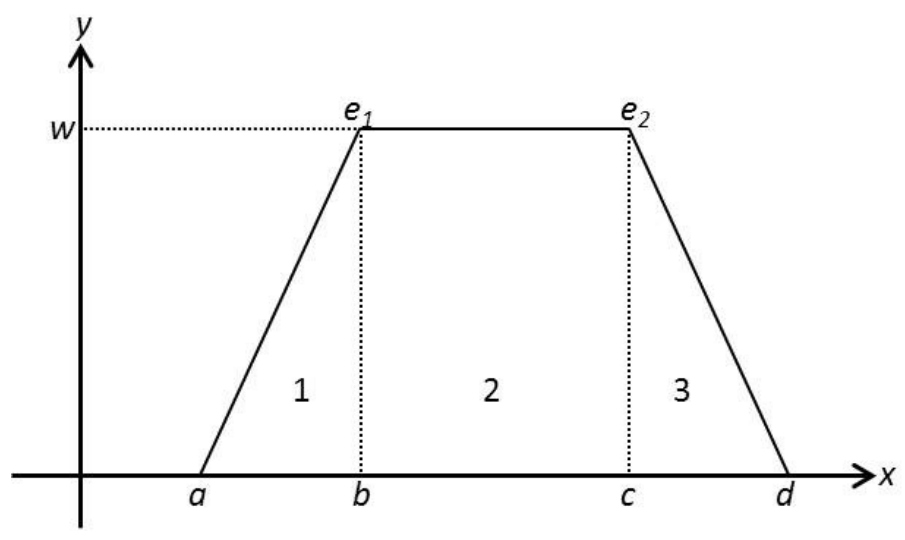

Figure 2: Trapezoidal fuzzy number.

\section{Convergence system, SC}

This section is based on [5]. Let:

1. $0 \leq f_{1}, f_{2}, f_{3} \leq 1$,

2. $f_{1}+f_{2}+f_{3}=1$,

3. $0<w \leq 1$,

4. $a_{i} \in \mathbb{R}, \alpha_{i} \in \mathbb{R}$.

Consider the following class of fuzzy numbers:

$C(\tilde{\mathbf{B}})=\left\{\tilde{B}_{i}=\left(a_{i}, f_{1}, f_{2}, f_{3}, \alpha_{i} ; w\right) \mid\right.$ satisfying the preceding four conditions $\}$, where:

1. $b_{i}=a_{i}+f_{1} \alpha_{i}$

2. $c_{i}=a_{i}+\left(f_{1}+f_{2}\right) \alpha_{i}$;

3. $d_{i}=a_{i}+\left(f_{1}+f_{2}+f_{3}\right) \alpha_{i}=a_{i}+\alpha_{i}$.

Property 1 For any two fuzzy numbers in $\tilde{B}_{1}, \tilde{B}_{2} \in C(\tilde{\mathbf{B}})$, such that $\tilde{B}_{1}=$ $\left(a_{1}, f_{1}, f_{2}, f_{3}, \alpha_{1} ; w\right)$ and $\tilde{B}_{2}=\left(a_{2}, f_{1}, f_{2}, f_{3}, \alpha_{2} ; w\right)$ and $\forall c \in \mathbb{R}$, satisfied:

1. $\tilde{B}_{1} c=\left(c a_{1}, f_{1}, f_{2}, f_{3}, c \alpha_{1} ; w\right)$.

2. $\tilde{B}_{1}+\tilde{B}_{2}=\left(a_{1}+a_{2}, f_{1}, f_{2}, f_{3}, \alpha_{1}+\alpha_{2} ; w\right)$. 
Definition 1 Given a function $G: C(\tilde{\mathbf{B}}) \longrightarrow \mathbb{R}$, and $\tilde{B}_{1}, \tilde{B}_{2} \in C(\tilde{\mathbf{B}})$ it is said that they are $S C$-equivalent if and only if:

$$
G\left(\tilde{B}_{1}\right)=G\left(\tilde{B}_{2}\right)
$$

Definition 2 Given a function $G: C(\tilde{\mathbf{B}}) \longrightarrow \mathbb{R}$, and a $g_{G} \in \mathbb{R}$, in the codomain of $G$, the following $S C$-equivalence class is defined $\tilde{\mathbf{B}}_{g_{G}} \subset C(\tilde{\mathbf{B}})$ as:

$$
\tilde{\mathbf{B}}_{g_{G}}=\left\{\tilde{B} \in C(\tilde{\mathbf{B}}) \mid G(\tilde{B})=g_{G}\right\} .
$$

\section{Comments:}

1. $\tilde{\mathbf{B}}_{g_{G}}$ is an equivalence class.

2. $\tilde{\mathbf{B}}_{g_{1}} \cap \tilde{\mathbf{B}}_{g_{2}}=\emptyset, \forall g_{1} \neq g_{2}$

3. $\bigcup_{g \in \mathbb{R}} \tilde{\mathbf{B}}_{g}=C(\tilde{\mathbf{B}})$.

Definition 3 Let $f_{1}, f_{2}, f_{3}, w$ such that they satisfy:

1. $0 \leq f_{1}, f_{2}, f_{3} \leq 1$,

2. $f_{1}+f_{2}+f_{3}=1$,

3. $0<w \leq 1$.

We define:

1. $F_{1}=\left(1+f_{2}\right)$.

2. $F_{2}=\left(2 f_{1}^{2}+6 f_{1} f_{2}+3 f_{2}^{2}+3 f_{3}-2 f_{3}^{2}\right)$.

3. $F_{3}=\left(3 f_{1}^{3}+4 f_{2}^{3}+3 f_{3}^{3}+12 f_{1} f_{2}^{2}+12 f_{2} f_{1}^{2}+6 f_{3}-8 f_{3}^{2}\right)$, It can be proven that $F_{3}>0$.

4. $A=\frac{12}{w^{3}\left(1+3 f_{2}\right)}$.

Theorem 1 Let:

$$
G: C(\tilde{\mathbf{B}}) \longrightarrow \mathbb{R}
$$

such that:

$$
G(\tilde{B})=A\left[\alpha^{2} F_{3}-4 \alpha F_{2}+6 a F_{1}\right] .
$$

Then $G$ is bijective on each $S C$-equivalence class.

Proof. See [5], Appendix A. 
Proposition 1 Let $\tilde{B} \in C(\tilde{\mathbf{B}}), s^{*}, s, \epsilon \in \mathbb{R}$ such that $s=s^{*}+\epsilon$, then:

$$
\lim _{\epsilon \rightarrow 0} G(\tilde{B s})=G\left(\tilde{B s^{*}}\right)
$$

Proof. See [5], Appendix B.

Proposition 2 Let $\tilde{B}_{i} \in C(\tilde{\mathbf{B}})$, and $s_{i}, \epsilon_{i} \in \mathbb{R}$ such that $s_{i}=s_{i}^{*}+\epsilon_{i}$, $i=1,2, \ldots, n$

$$
\lim _{\left\{\epsilon_{i}\right\}_{i=1}^{n} \rightarrow 0} G\left(\sum_{i=1}^{n} \tilde{B}_{i} s_{i}\right)=G\left(\sum_{i=1}^{n} \tilde{B}_{i} s_{i}^{*}\right) .
$$

Proof. See [5], Appendix C.

Definition 4 Consider the following multiobjective optimization problem $h(x)$, $x \in X \subseteq \mathbb{R}^{n}$, where:

$$
h(x)=\left(h_{1}(x), h_{2}(x), \ldots, h_{k}(x)\right) ; h_{i}: \Re^{n} \rightarrow \mathbb{R}, i=1,2, \ldots, k .
$$

Then:

1. Given $y=\left(y_{1}, y_{2}, \ldots, y_{k}\right)$ is said that it dominates $z=\left(z_{1}, z_{2}, \ldots, z_{k}\right)$ if and only if $\forall i \in\{1,2, \ldots, k\} y_{i} \leq z_{i}$ and $\exists i_{0} \in\{1,2, \ldots, k\}$ such that $y_{i_{0}}<z_{i_{0}}$.

2. Pareto Frontier $(P F)=\{h(x) \mid$ are non dominated $\}$.

3. A solution vector $x^{*}$ is said to be Pareto Optimal if and only if there is no other vector $x$ such that $h(x)$ dominates $h\left(x^{*}\right)$.

Definition 5 The SC-Frontier (SC-F) is defined as:

$S C-F=\{h(x) \mid \forall \epsilon>0 \exists y \in C o d o m i n e(h)$-Image $(h)$ such that $\|y-h(x)\|<\epsilon\}$.

Proposition $3 P F \subseteq S C-F$.

Proof. See [5], Appendix D.

Definition 6 Given a multiobjective optimization problem $h(x), \quad x \in X \subseteq \mathbb{R}^{n}$, where:

$$
h(x)=\left(h_{1}(x), h_{2}(x), \ldots, h_{k}(x)\right) ; h_{i}: \Re^{n} \rightarrow \mathbb{R}, i=1,2, \ldots, k,
$$


and

$$
\tilde{\mathbf{B}}=\left(\tilde{B}_{1}, \tilde{B}_{2}, \ldots, \tilde{B}_{k}\right)
$$

where $\tilde{B}_{i} \in C(\tilde{\mathbf{B}}), i=1, \ldots, k$. Define:

$$
G(\tilde{\mathbf{B}} h(x))=G\left(\sum_{i=1}^{k} \tilde{B}_{i} h_{i}(x)\right)
$$

Definition 7 Given a multiobjective optimization problem and $a \tilde{\mathbf{B}}$, define:

$$
x_{\tilde{B}}^{*}=\operatorname{argmin}\{G(\tilde{\mathbf{B}} h(x)) \mid h(x) \in S C-F\} .
$$

Definition 8 The optimal set of $S C-(S C-O)$ solutions is defined as:

$$
S C-O=\left\{x_{\tilde{B}}^{*}, \tilde{B} \in C(\tilde{\mathbf{B}})\right\} .
$$

\subsection{Empirical boundary conditions}

Given an optimization problem with $k$ objective functions, the following values have been used empirically:

1. $f_{1}=f_{3}=0.25, f_{2}=0.5, w=1$,

2. $\alpha_{i} \in\left[-12 K_{1}, 12 K_{1}\right], i=1,2, \ldots, k$,

3. $a_{i}=K_{2}+K_{3} g_{i}$,

where: $K_{1}=\frac{2 F_{2}}{F_{3}}, K_{2}=\frac{2 F_{2}}{3 k F_{1}}, K_{3}=\frac{A^{-1} F_{3}}{6 k F_{1}}, g_{i}=\left\{\begin{array}{lll}1 & \text { if } & h_{i}\left(x^{*}\right)>0 \\ 0 & \text { if } & h_{i}\left(x^{*}\right)=0 \\ -1 & \text { if } & h_{i}\left(x^{*}\right)<0\end{array}\right.$

\subsection{SC algorithm}

\section{Step 0:}

1. Provide stopping criteria

2. Set $f_{1}, f_{2}, f_{3}, w$ such that they satisfy the conditions of Theorem 1

3. Given the function to optimize $h=\left(h_{1}, h_{2}, \ldots h_{k}\right)$, find the boundary conditions (or use the suggested empirical conditions), take $\tilde{B}_{i} \in C(\tilde{\mathbf{B}})$, $i=1,2, \ldots, k$.

4. Set values for $a_{i}$ and $\alpha_{i}$. 
5. Take any value $x$ in the Domain of $h$ and evaluate:

$$
G(\tilde{B} h(x))=G\left(\sum_{i=1}^{k} \tilde{B}_{i} h_{i}(x)\right)
$$

Set:

$$
g_{G}(x) \leftarrow G(\tilde{B} h(x)) .
$$

Step 1: Through $m$ neighborhoods, find $x_{j} \in \operatorname{Phase}^{j}(x)$ and evaluate $G\left(\tilde{B} h\left(x_{j}\right)\right), j=1,2, \ldots, m$

$$
\begin{gathered}
g_{G}\left(x_{1}\right)=\min _{j}\left\{G\left(\tilde{B} h\left(x_{j}\right)\right), G(\tilde{B} h(x))\right\}, \\
x \leftarrow x_{1} .
\end{gathered}
$$

Step 2: Stop in the following conditions:

1. If for a certain number of iterations $g_{G}\left(x_{1}\right)=g_{G}(x)$, then an optimum was reached.

2. Stop criteria is met.

In any other case go to Step 1.

Remark: when $g_{G}$ is very close to zero, it can be used, for example: $\left(1-g_{G}\right)^{2}$.

\section{Particle swarm optimization (PSO)}

The particle swarm optimization is a metaheuristic based on swarm intelligence and has its roots in artificial life, social psychology, engineering and computer science. PSO differs from evolutionary computation (c.f. [20]) because the population members or agents, also called particles, are "flying" through the problem hyperspace.

PSO is an adaptive method that uses agents or particles moving through the search space using the principles of evaluation, comparison and imitation [20].

PSO is based on the use of a set of particles or agents that correspond to states of an optimization problem, where each particle moves across the solution space in search of an optimal position or at least a good solution. In PSO, agents communicate with each other, and the agent with the best position (measured according to an objective function) influences the others by attracting them towards itself. 
The population is initialized by assigning a random position and speed to each agent. At each iteration, the velocity of each particle is randomly accelerated towards its best position (where the value of the fitness function or objective function improves) and also considering its neighbors' best position.

To solve a problem, PSO uses a dynamic management of particles; this approach allows breaking cycles and diversifying the search. In this work, a $r$ particle swarm is represented at time $t$ under the form:

$$
\theta_{1 t}, \theta_{2 t}, \ldots, \theta_{r t}
$$

with $\theta_{j t} \in D, j=1,2, \ldots, r$, then a movement of the swarm is defined according to equation 1 .

$$
\theta_{j t+1}=\theta_{j t}+V_{j t+1}
$$

where the velocity $V_{j t+1}$ is given in equation 2

$$
V_{j, t+1}=\alpha V_{j, t}+\operatorname{rand}\left(0, \varphi_{1}\right)\left[\theta_{j, t}^{\prime}-\theta_{j, t}\right]+\operatorname{rand}\left(0, \varphi_{2}\right)\left[\theta_{g, t}^{\prime}-\theta_{j, t}\right],
$$

where:

$D$ : space of feasible solutions,

$V_{j, t}:$ speed at time $t$ of the $j$-th particle,

$V_{j, t+1}$ : speed at time $t+1$ of the $j$-th particle,

$\theta_{j, t}: j$-th particle at time $t$,

$\theta_{g, t}^{\prime}:$ the particle with the best value found so far (i.e., before time $t$ ),

$\theta_{j, t}^{\prime}:$ best position found so far by the $j$-th particle (before time $t$ ),

$\operatorname{rand}(0, \varphi)$ : random number uniformly distributed over the interval $[0, \varphi]$,

$\alpha$ : inertia weight factor.

The PSO algorithm is described in Table 1 . 
Table 1: PSO algorithm.

1. Create a population of particles distributed in the feasible space.

2. Evaluate each position of the particles according to the objective function (fitness function).

3. If the current position of a particle is better than the previous one, update it.

4. Determine the best particle (according to the best previous positions).

5. Update the particle velocities $j=1,2, \ldots, r$ according to equation 2 .

6. Move the particles to new positions according to equation 1 .

7. Go to Step 2 until the termination criterion is satisfied.

\subsection{SC-PSO-3P}

The three-phase PSO algorithm along with the SC criterion is named SC-PSO3P. The SC-PSO-3P algorithm is described in Table 1 .

As can be seen in the algorithm in table 1 , the key modification is to consider the SC criterion, and the 3 phases, the rest of the algorithm remains unchanged. It should be mentioned, that any other search algorithm can be used instead of PSO, although the respective tests must be performed.

We describe the main characteristics of the proposed algorithm, called PSO3P. This algorithm is based in a traditional PSO heuristic. However, the position of the particles can be modified using different strategies, that are applied in different times, or phases, of the searching process.

In phase 1, called stabilization, according to the description presented in section 4, the PSO-3P algorithm generates randomly a set of particles in the solution space. Then, during $i t F_{1}$ iterations the position of the particles is modified using equations (1) and (2). Thus, at the end of this phase the particles are concentrated, or stabilized, in a promising region.

When phase 1 is completed, a breadth-first search strategy, called phase 2, is incorporated. In this phase, if the global best solution is not improved after three consecutive iterations, the position of $M_{2}$ particles is modified randomly. However, the particle with the best known position is preserved. Thus, the population is dispersed in the solution space, but it can be attracted to the best region visited so far. This diversification strategy is considered during $i t F_{2}$ iterations.

Finally, phase 3 is initialized. During $i t F_{3}$ iterations the following depth-first 
search strategy is applied. If the global best solution is not improved after three consecutive iterations, $M_{3}$ particles are randomly positioned in a neighborhood of the best known solution. Thus, phase 3 includes an intensification process in a promising region.

The algorithm was implemented in Matlab R2008a and was run in an Intel Core i5-3210M processor computer at $2.5 \mathrm{GHz}$, running on Windows 8.

\section{Computational results}

The parameters were set as follows, $\phi_{1}=\phi_{2}=2.05$, and $\phi=\phi_{1}+\phi_{2}$, then:

$$
\alpha=\frac{2}{\phi-2+\sqrt{\phi^{2}-4 * \phi}} ; \quad \varphi_{1}=\alpha * \phi_{1} ; \quad \varphi_{2}=\alpha * \phi_{2} .
$$

For phase 2 and phase 3 the parameters were set as $c=3$, it $F_{1}=75, i t F_{2}=$ $150, i t F_{3}=$ MaxIt, prop $=0.25$.

The select problems have been well studied before as benchmarks by various approaches. The 24 constrained problems(G1-G24) have been solved before using the following algorithms : self-adaptive multi-strategy differential evolution with local search(Memetic-SAMSDE) [7], adaptive penalty formulation with GA(APF-GA) [32], modified differential evolution(MDE) [29], adapted constrained particle swarm optimization(CVI-PSO) [27], a differential evolution combined variants (DECV) [29] and a heuristic inspired by T-Cell model of the immune system (T-CELL) [1].

The three engineering problems considered are a pressure vessel design problem(eng01) [18], a welded beam design problem(eng02) [30] and a tensioncompression string design problem(eng03) [2]. Eng01 and eng03, have been solved by the following approaches: constraint violation with interval arithmetic PSO(CVI-PSO) [27], a co-evolutionary PSO(CPSO) [11], a hybrid PSO(HPSO) [12], an accelerating technique(AATM) [35], a T-Cell heuristic [1]. Problem eng02 has been solved by these approach: CVI-PSO, a GA with binary representation (GAPF) [4], a GA based co-evolution model (CGA) [3], CPSO and HPSO.

In this section the results obtained for constrained optimization will be presented, in order to show the efficiency of SC. A study in depth for multiobjective optimization, and unconstrained optimization, will be presented in future work.

In this paper, the functions for restricted optimization were taken from [22]. 


\section{Begin}

while Termination criterion is not satisfied do

Set variables $c, i t F_{1}, i t F_{2}, i t F_{3}$, MaxIt and prop.

Create a population of $n$ Pop random particles.

Set $c o n t=0$ and $i t=1$. Evaluate each position of the particles according to the SC function.

If the current position of a particle is better (respect to the fitness function) than the previous update it.

Determine the best particle (according to the best previous positions against the optimization criterion). If a better particle cannot be founded, let cont $=$ cont +1 .

Update the particle velocities $j=1,2, \ldots, n$ Pop according to equation (2).

(Phase 1: Stabilization) if $i t \leq i t F 1$ then | go to Step 34.

end

(Phase 2: Breadth-first search) if $i t F 1<i t \leq i t F 2$ then

if cont $=c$ then

Set $\mathrm{n}=1$. while $n \leq n P o p *$ prop do

Create a random particle and, with a probability bigger

than 0.5 substitute randomly a particle in the swarm.

Set $n=n+1$.

end

Set cont $=0$.

end

go to Step 34.

end

end

(Phase 3: Depth-first search). if $i t F 2<i t \leq i t F 3$ then

if cont $=c$ then

Set $n=1$. while $n \leq n P o p *$ prop. do

Create a random particle in a variable neighborhood of $\theta_{g, t}^{\prime}$ and substitute randomly a particle in the swarm. Set $n=n+1$.

end

end

Set $\operatorname{cont}=0$.

go to Step 34 .

end

Select the best $n P o p$ particles according to SC optimization criterion.

Set $i t=i t+1$. Go to Step 3 until the termination criterion is satisfied.

Algorithm 1: SC-PSO-3P algorithm. 


\subsection{Constrained optimization}

In this section we consider the global optimization problem with restrictions:

$$
\begin{array}{lrl}
\text { Minimize: } & h(x) \\
\text { subject to: } & f_{i}(x) \leq 0 & i=1,2, \ldots, m \\
& t_{j}(x)=0 & j=1,2, \ldots, k \\
& x \in D \subseteq S=[L, U]
\end{array}
$$

where:

- $[L, U]=\left\{x=\left(x_{1}, x_{2}, \ldots, x_{n}\right) \mid l_{i} \leq x_{i} \leq u_{i}\right\} \subset \mathbb{R}^{n}$.

- the feasible region $(D)$ is defined as:

$$
D=\left\{x \in S, f_{i}(x) \leq 0, \quad i=1,2, \ldots, m, t_{j}(x)=0, j=1,2, \ldots, k\right\} .
$$

The problem given in (3) can be transformed into the problem (4) [24] given by:

$$
\begin{array}{rlrl}
\text { Minimize }_{x \in D}: & F(x) & =\left(h_{1}(x), h_{2}(x), h_{3}(x)\right) \\
\text { with: } & h_{1}(x)=h(x) \\
& h_{2}(x)=P_{1} \sum_{i=1}^{m} \frac{c_{i}(x)}{c(x)+\epsilon} \\
& h_{3}(x)=P_{2} \sum_{j=1}^{k} d_{j}
\end{array}
$$

where:

- $c_{i}(x)=\max \left\{0, f_{i}(x)\right\} \cdot i=1,2, \ldots, m$.

- $c(x)=\max _{i=1,2, \ldots, m}\left\{c_{i}(x)\right\}$.

- $d_{j}(x)=\max \left\{0,\left|t_{j}(x)\right|-\delta\right\} \cdot j=1,2, \ldots, k$.

- $\epsilon, \delta>0, P_{i}>>0, i=1,2$.

In this section, the efficiency is defined as:

$$
\text { efficiency }=\left\{\begin{array}{lll}
1-a b s\left(\frac{h(x)-h\left(x^{*}\right)}{h\left(x^{*}\right)}\right) & \text { if } \quad h\left(x^{*}\right) \neq 0 \\
1-a b s(h(x)) & \text { if } \quad h\left(x^{*}\right)=0 .
\end{array}\right.
$$

Table 2 shows the ratio $(\rho)$ for problems G1 to G24, as well as the engineering problems eng01-eng03 and a summary of some characteristics of these problems [27]. 


\begin{tabular}{l|cc}
\hline \hline Function(n) & Type of $h$ & Rati* $\rho \%$ \\
\hline G1(13) & Quadratic & 0.0001 \\
G2(20) & Non-linear & 99.996 \\
G3(12) & Polynomial & 0.0000 \\
G4(5) & Quadratic & 26.925 \\
G5(4) & Cubic & 0.0000 \\
G6(2) & Quadratic & 0.0054 \\
G7(10) & Quadratic & 0.0002 \\
G8(2) & Non-linear & 0.8611 \\
G9(7) & Polynomial & 0.5314 \\
G10(8) & Linear & 0.0002 \\
G11(2) & Quadratic & 0.0000 \\
G12(3) & Quadratic & 4.5420 \\
G13(5) & Non-linear & 0.0000 \\
G14(10) & Non-linear & 0.0000 \\
G15(3) & Quadratic & 0.0000 \\
G16(5) & Non-linear & 0.0186 \\
G17(6) & Non-linear & 0.0000 \\
G18(9) & Quadratic & 0.0000 \\
G19(15) & Non-linear & 35.548 \\
G20(24) & Linear & 0.0000 \\
G21(7) & Linear & 0.0000 \\
G22(22) & Linear & 0.0000 \\
G23(9) & Linear & 0.0000 \\
G24(2) & Linear & 74.268 \\
\hline eng01(4) & Non-linear & 39.737 \\
eng02(4) & Non-linear & 2.6638 \\
eng03(3) & Non-linear & 0.7560 \\
\hline \hline & &
\end{tabular}

Table 2: Summary ([27]), $* \rho=\frac{\text { number of solution } \in D}{\text { number of solution } \in S}$. 


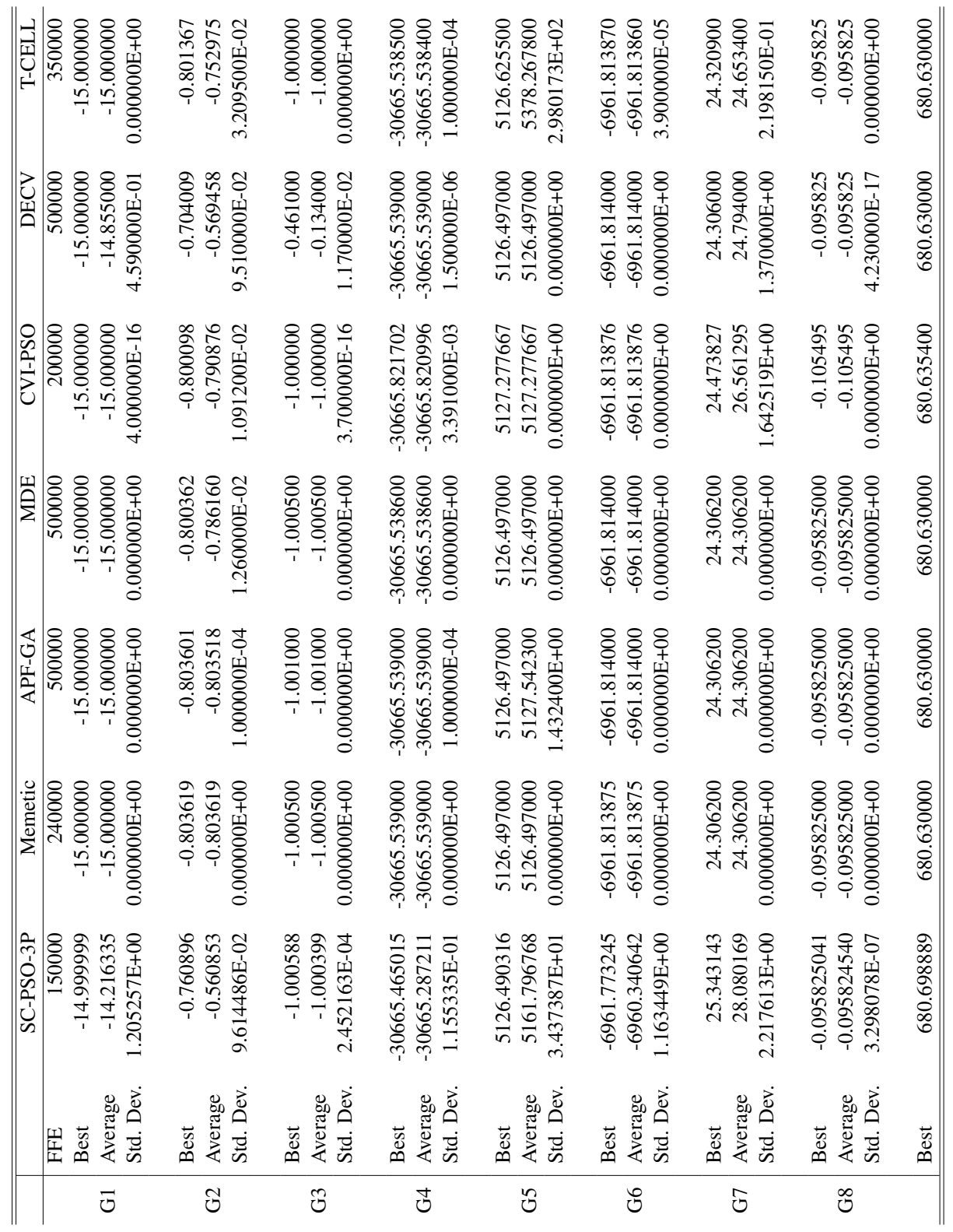

Table 3: Comparative results for several algorithms. 


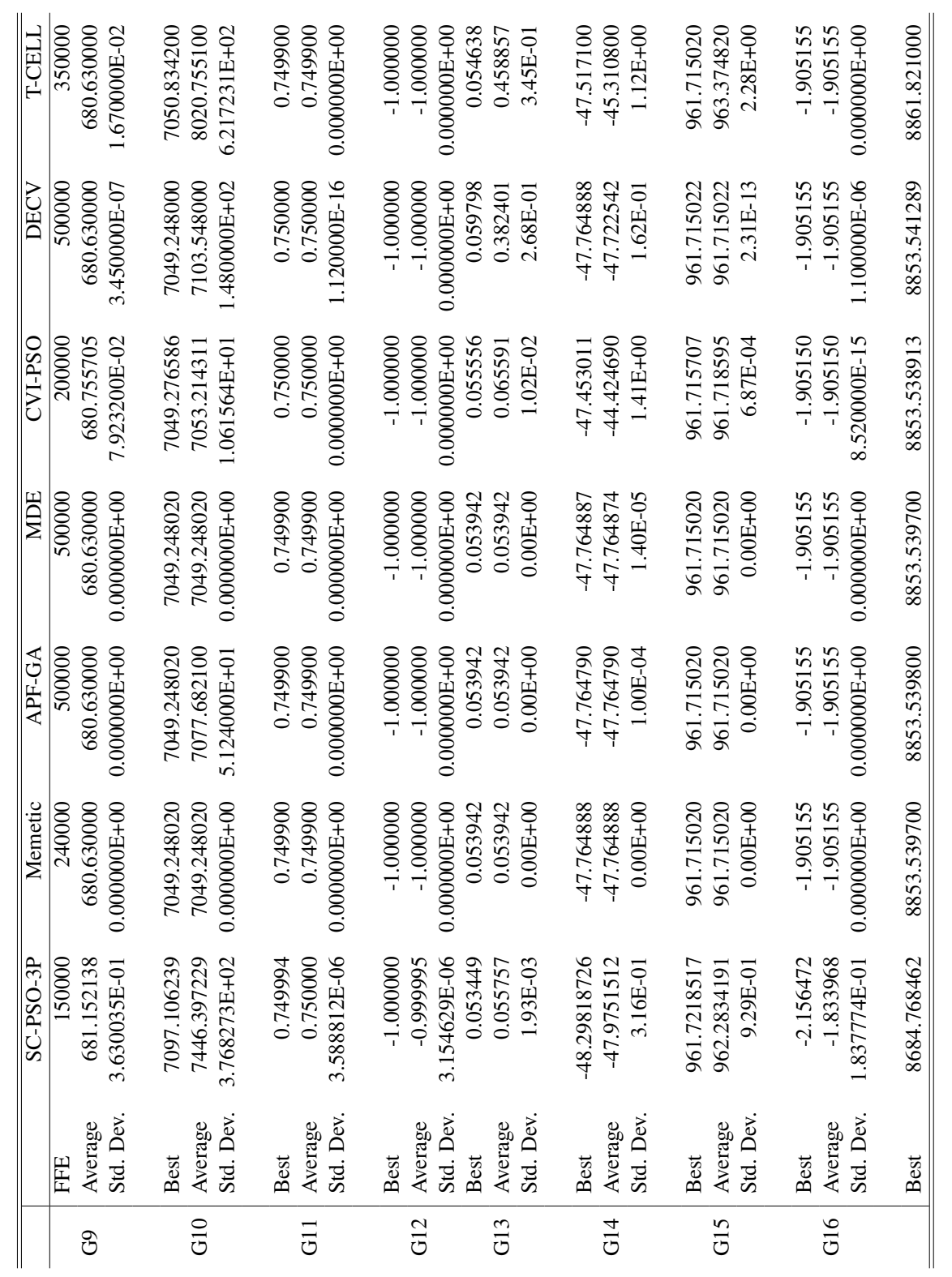

Table 4: Comparative results for several algorithms. 


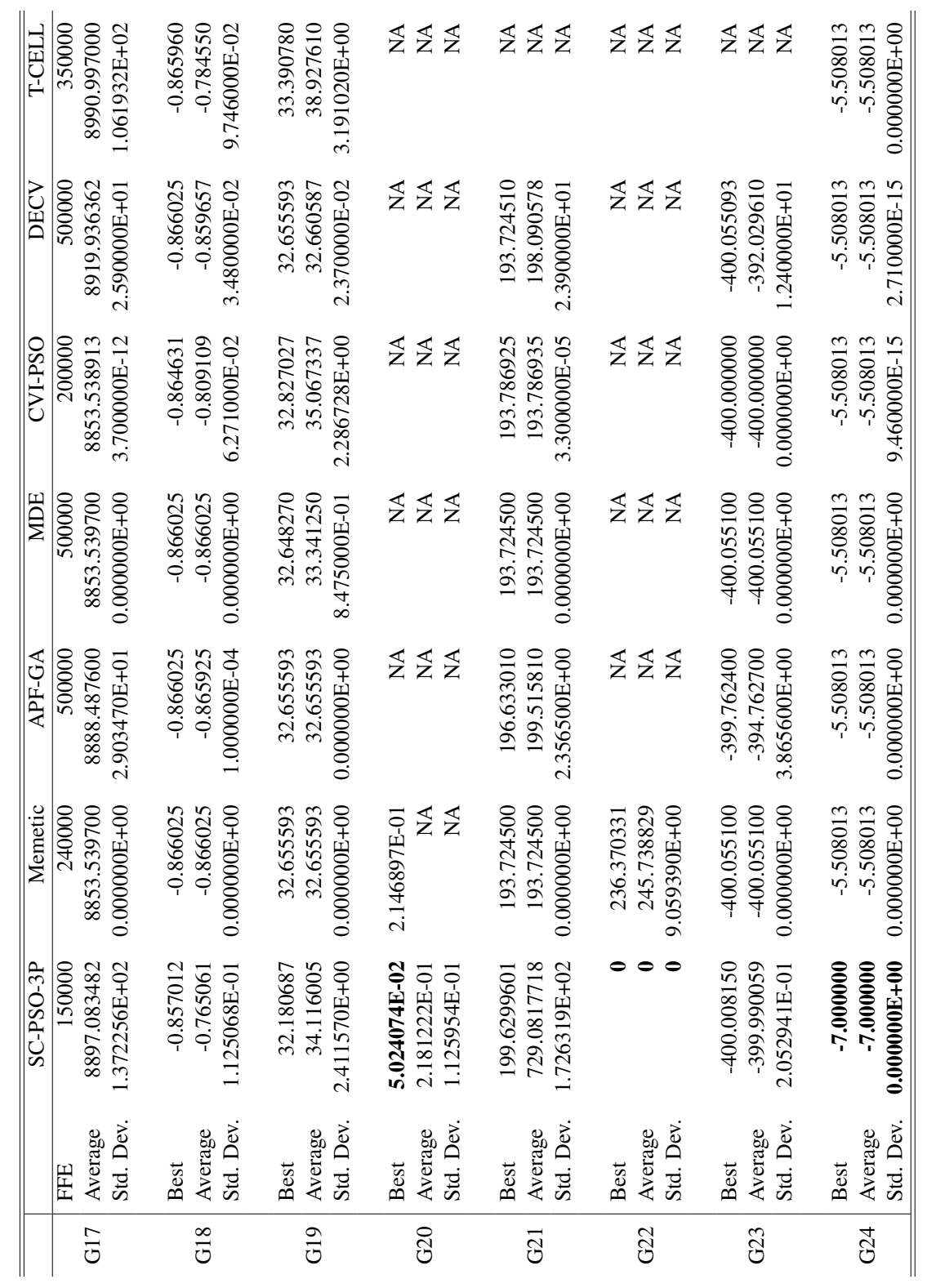

Table 5: Comparative results for several algorithms. 
Tables 3,4 and 5 show that the best solutions obtained by SC-PSO-3P are competitive with those achieved by other methods. In particular, for the problem G24 a better feasible solution was consistently reached as can be seen in Table 6. It is noteworthy that for this particular problem, in all runs the solution was reached in the first iteration.

\begin{tabular}{l|ll}
\hline \hline & best known & SC-PSO-3P \\
\hline$h\left(x^{*}\right)$ & -5.5080132771 & $\mathbf{- 7 . 0 0 0 0 0 0 0 0 0}$ \\
$x_{1}^{*}$ & 2.39520197477613 & 3 \\
$x_{2}^{*}$ & 3.178493074117714 & 4 \\
$f_{1} \leq 0$ & $-1.7763568394 \mathrm{e}-15$ & -160.00 \\
$f_{2} \leq 0$ & 0.00 & -152.00 \\
\hline \hline
\end{tabular}

Table 6: Table comparing the best known solution vs. SC-PSO-3P in G24.

From literature it is known that regarding the function G20 there is not a single feasible solution while for the function G22, a feasible solution is rarely found [22], for this reason is very common that these instances are not considered when they propose some method. However, it may be noticed that SC-PSO$3 \mathrm{P}$ is competitive results even consider that improves the results reported in the literature as can be seen from tables 7 and 8 ,

For instance G20, in [22] is said "This solution is a little unfeasible and no feasible solution is found so far":

[22] G20:

$x^{*}=(1.28582343498528086 \mathrm{E}-18,4.8346030252613066 \mathrm{E}-34,0$,

$0,6.30459929660781851 \mathrm{E}-18,7.57192526201145068 \mathrm{E}-34$,

$5.0335069837240437 \mathrm{E}-34,9.282680796166 \mathrm{E}-34,0$,

$1.76723384525547359 \mathrm{E}-17,3.55686101822965701 \mathrm{E}-34$,

2.99413850083471346E-34, 0.158143376337580827,

$2.29601774161699833 \mathrm{E}-19,1.06106938611042947 \mathrm{E}-18$,

$1.31968344319506391 \mathrm{E}-18,0.530902525044209539,0$,

$0.289148310257773535 \mathrm{E}-18,3.34892126180666159 \mathrm{E}-18,0$,

$0.310999974151577319,5.41244666317833561 \mathrm{E}-5$,

4.84993165246959553E-16);

$h^{*}=204.979400285636 \mathrm{e}-003$.

With SC several improved solutions. Below are two of them:

SC-PSO-3P (Example 1) G20:

$x^{*}=(1.00 \mathrm{E}-300,1.00 \mathrm{E}-300,1.00 \mathrm{E}-300,1.00 \mathrm{E}-300$, 
$1.00 \mathrm{E}-300,1.00 \mathrm{E}-300,1.00 \mathrm{E}-300,1.00 \mathrm{E}-300,1.00 \mathrm{E}-300$, $1.00 \mathrm{E}-300,1.00 \mathrm{E}-300,0.296470402,1.00 \mathrm{E}-300,1.00 \mathrm{E}-300$, $1.00 \mathrm{E}-300,1.00 \mathrm{E}-300,1.00 \mathrm{E}-300,1.00 \mathrm{E}-300,1.00 \mathrm{E}-300$, $1.00 \mathrm{E}-300,1.00 \mathrm{E}-300,1.00 \mathrm{E}-300,1.00 \mathrm{E}-300,0.703529139)$; $h^{*}=8.999996 \mathrm{E}-02$.

SC-PSO-3P (Example 2) G20:

$x^{*}=(1.00 \mathrm{E}-300,1.00 \mathrm{E}-300,1.00 \mathrm{E}-300,1.00 \mathrm{E}-300$, $1.00 \mathrm{E}-300,1.00 \mathrm{E}-300,1.00 \mathrm{E}-300,1.00 \mathrm{E}-300,1.00 \mathrm{E}-300$, $1.00 \mathrm{E}-300,1.37 \mathrm{E}-06,1.00 \mathrm{E}-300,1.00 \mathrm{E}-300,1.00 \mathrm{E}-300$, $1.00 \mathrm{E}-300,1.00 \mathrm{E}-300,1.00 \mathrm{E}-300,1.00 \mathrm{E}-300,1.00 \mathrm{E}-300$, $1.00 \mathrm{E}-300,1.00 \mathrm{E}-300,1.00 \mathrm{E}-300,0.502406032,1.00 \mathrm{E}-300)$; $h^{*}=50.24074020 \mathrm{e}-003$.

\begin{tabular}{l|cc}
\hline \hline$[22]$ & SC-PSO-3P (Example 1) & SC-PSO-3P (Example 2) \\
\hline $2.049794 \mathrm{E}-01$ & $\mathbf{5 . 0 2 4 0 7 4 E - 0 2}$ & $\mathbf{8 . 9 9 9 9 9 6 E - 0 2}$ \\
$f(x) \leq 0$ & & \\
$1.317752 \mathrm{E}-01$ & $2.847350 \mathrm{E}-300$ & $1.666667 \mathrm{E}-300$ \\
$1.766032 \mathrm{E}-19$ & $2.492499 \mathrm{E}-300$ & $1.538462 \mathrm{E}-300$ \\
$7.578526 \mathrm{E}-19$ & $2.216294 \mathrm{E}-300$ & $1.428572 \mathrm{E}-300$ \\
$2.224047 \mathrm{E}-19$ & $2.492499 \mathrm{E}-300$ & $1.538462 \mathrm{E}-300$ \\
$2.092945 \mathrm{E}-18$ & $1.814211 \mathrm{E}-300$ & $1.250000 \mathrm{E}-300$ \\
$0.000000 \mathrm{E}+00$ & $2.492499 \mathrm{E}-300$ & $1.538462 \mathrm{E}-300$ \\
$t(x)=0$ & & \\
$1.000000 \mathrm{E}-04$ & $-2.358454 \mathrm{E}-294$ & $-1.227952 \mathrm{E}-299$ \\
$-2.197257 \mathrm{E}-17$ & $-4.585322 \mathrm{E}-295$ & $-1.294290 \mathrm{E}-300$ \\
$1.553971 \mathrm{E}-18$ & $-6.610392 \mathrm{E}-295$ & $-2.515003 \mathrm{E}-300$ \\
$8.175374 \mathrm{E}-19$ & $-8.994252 \mathrm{E}-296$ & $7.952623 \mathrm{E}-302$ \\
$1.000000 \mathrm{E}-04$ & $-5.889714 \mathrm{E}-295$ & $-2.843776 \mathrm{E}-300$ \\
$-1.038308 \mathrm{E}-17$ & $-1.362617 \mathrm{E}-295$ & $-3.215523 \mathrm{E}-301$ \\
$-3.346898 \mathrm{E}-17$ & $-6.685073 \mathrm{E}-295$ & $-2.663043 \mathrm{E}-300$ \\
$-3.208538 \mathrm{E}-18$ & $-7.027115 \mathrm{E}-296$ & $5.820753 \mathrm{E}-301$ \\
$0.000000 \mathrm{E}+00$ & $-1.323114 \mathrm{E}-296$ & $5.604647 \mathrm{E}-301$ \\
$1.000000 \mathrm{E}-04$ & $-1.803505 \mathrm{E}-295$ & $-5.182852 \mathrm{E}-302$ \\
$1.000000 \mathrm{E}-04$ & $9.787500 \mathrm{E}-01$ & $1.760682 \mathrm{E}-300$ \\
$6.866522 \mathrm{E}-16$ & $-8.951397 \mathrm{E}-297$ & $9.840000 \mathrm{E}-01$ \\
$1.000000 \mathrm{E}-04$ & $4.975926 \mathrm{E}-01$ & $4.586120 \mathrm{E}-07$ \\
$1.000000 \mathrm{E}-04$ & $1.200004 \mathrm{E}-01$ & $1.433791 \mathrm{E}-07$ \\
\hline \hline
\end{tabular}

Table 7: Table comparing G20 [22] vs. SC-PSO-3P. 
In Table 7 a comparison of the values of the function and constraints is presented for the function G20. Two solutions found by SC-PSO-3P are provided, can be observed that both improve over the best reported value, however, example 2 is "less unfeasible" than example 1.

Regarding the problem G22, the best reported solution in the literature and the solution obtained by SC-PSO-3P are provided below.

[22] G22:

$x^{*}=(236.430975504001054,135.82847151732463,204.818152544824585$, 6446.54654059436416, 3007540.83940215595, 4074188.65771341929, 32918270.5028952882, 130.075408394314167, 170.817294970528621, 299.924591605478554, 399.258113423595205, 330.817294971142758, 184.51831230897065, 248.64670239647424, 127.658546694545862, 269.182627528746707, 160.000016724090955, 5.29788288102680571, 5.13529735903945728, 5.59531526444068827, 5.434444779314453499, 5.07517453535834395);

$h^{*}=236.430975504001 \mathrm{e}+000$.

With SC-PSO-3P an improved solution:

SC G22:

$x^{*}=(0,0,0,0,0,0,0,100,100,100.010,100,100,101.9292514$,

$0,13.653013870,100.00034450,0.010,-4.700,-2.7379525440$,

4.6039351080, $-0.7642880070,-2.7215211340)$;

$h^{*}=0.0000 \mathrm{E} 00$.

In Table 8 a comparison of the values of the function and constraints is presented for the function G22.

Regarding the engineering problems eng01-eng03, the results obtained by various methods are shown in Tables 9,11 and 12. We can see that for the pressure vessel design problem, in Table 10 a better feasible solution was found with respect to those reported previously. For the other two problems, the results obtained by SC-PSO-3P are very competitive over other proposed algorithms.

In Table 13, results of the bootstrap test are shown. 


\begin{tabular}{l|c}
\hline \hline$[22]$ & SC-PSO-3P \\
\hline$h^{*}=2.36 \mathrm{E}+02$ & $h^{*}=\mathbf{0 . 0 0 E}+\mathbf{0 0}$ \\
\hline$f_{1}(x) \leq 0$ & \\
$-2.21 \mathrm{E}-07$ & $0.00 \mathrm{E}+00$ \\
$t_{i}(x)=0$ & \\
$-2.93 \mathrm{E}-05$ & $0.00 \mathrm{E}+00$ \\
$9.20 \mathrm{E}-05$ & $0.00 \mathrm{E}+00$ \\
$-3.42 \mathrm{E}+07$ & $-6.00 \mathrm{E}+07$ \\
$-6.00 \mathrm{E}+07$ & $-4.30 \mathrm{E}+07$ \\
$-7.99 \mathrm{E}+07$ & $-5.40 \mathrm{E}+07$ \\
$-6.62 \mathrm{E}+07$ & $-7.60 \mathrm{E}+07$ \\
$5.61 \mathrm{E}-05$ & $0.00 \mathrm{E}+00$ \\
$1.67 \mathrm{E}-05$ & $0.00 \mathrm{E}+00$ \\
$5.41 \mathrm{E}-05$ & $0.00 \mathrm{E}+00$ \\
$\mathbf{4 . 0 7 E}+\mathbf{0 6}$ & $\mathbf{3 . 4 4 E - 0 4}$ \\
$1.67 \mathrm{E}-05$ & $1.00 \mathrm{E}-02$ \\
$5.74 \mathrm{E}-05$ & $9.48 \mathrm{E}-02$ \\
$5.74 \mathrm{E}-05$ & $8.04 \mathrm{E}+00$ \\
$7.48 \mathrm{E}-05$ & $1.24 \mathrm{E}-03$ \\
$7.47 \mathrm{E}-05$ & $6.47 \mathrm{E}+00$ \\
$-6.16 \mathrm{E}-07$ & $-1.88 \mathrm{E}+00$ \\
$6.12 \mathrm{E}-06$ & $-2.84 \mathrm{E}-05$ \\
$-8.78 \mathrm{E}-05$ & $3.00 \mathrm{E}+02$ \\
$9.59 \mathrm{E}-05$ & $-3.14 \mathrm{E}-02$ \\
\hline$\sum_{i} a b s\left(t_{i}\right)$ & \\
$2.44 \mathrm{E}+08$ & $2.33 \mathrm{E}+08$ \\
\hline \hline
\end{tabular}

Table 8: Table comparing G22 [22] vs. SC.

\begin{tabular}{l|ccccc}
\hline \hline & FFE & Best & Average & Worst & Std. Dev. \\
\hline SC-PSO-3P & 50000 & $\mathbf{5 8 9 8 . 5 4 9 3 8 6}$ & 6278.578324 & 7335.299154 & $2.551714 \mathrm{E}+02$ \\
CPSO & 200000 & 6061.077700 & 6147.133200 & 6363.804100 & $8.645450 \mathrm{E}+01$ \\
HPSO & 81000 & 6059.725500 & 6099.932300 & 6288.677000 & $8.620220 \mathrm{E}+01$ \\
AATM & 30000 & 6059.725500 & 6061.987800 & 6090.802200 & $4.700000 \mathrm{E}+00$ \\
T-Cell & 81000 & 6390.554000 & 6737.065100 & 7694.066800 & $3.570000 \mathrm{E}+02$ \\
CVI-PSO & 25000 & 6059.714300 & 6292.123100 & 6820.410100 & $2.884550 \mathrm{E}+02$ \\
\hline \hline
\end{tabular}

Table 9: Results of different algorithms for pressure vessel design problem. 


\begin{tabular}{l|rr}
\hline \hline & SC-PSO-3P & Best Known \\
\hline$h\left(x^{*}\right)$ & $\mathbf{5 8 9 8 . 5 4 9 3 8 6}$ & 6059.7143 \\
\hline$x_{1}^{*}$ & 0.778643603 & 0.8125 \\
$x_{2}^{*}$ & 0.38712201 & 0.4575 \\
$x_{3}^{*}$ & 40.33557909 & 42.0984456 \\
$x_{4}^{*}$ & 200 & 176.6365958 \\
\hline$f_{1} \leq 0$ & $-1.66926028 \mathrm{E}-04$ & $-1.05992992 \mathrm{E}-12$ \\
$f_{2} \leq 0$ & $-2.32058574 \mathrm{E}-03$ & $-3.58808290 \mathrm{E}-02$ \\
$f_{3} \leq 0$ & $-1.13500617 \mathrm{E}+03$ & $6.23567029 \mathrm{E}-06$ \\
$f_{4} \leq 0$ & $-4.00000000 \mathrm{E}+01$ & $-6.33634042 \mathrm{E}+01$ \\
\hline \hline
\end{tabular}

Table 10: Results comparative pressure vessel problem best known vs. SC-PSO-3P.

\begin{tabular}{l|ccccc}
\hline \hline & FFE & Best & Average & Worst & Std. Dev. \\
\hline SC-PSO-3P & 50000 & 1.724958 & 1.738032 & 1.843013 & $2.555789 \mathrm{E}-02$ \\
GAPF & NA & 2.433116 & NA & NA & NA \\
CGA & 900000 & 1.748309 & 1.771973 & 1.785835 & $1.122000 \mathrm{E}-02$ \\
CPSO & 200000 & 1.728024 & 1.748831 & 1.782145 & $1.292600 \mathrm{E}-02$ \\
HPSO & 81000 & 1.724852 & 1.749040 & 1.814295 & $4.004900 \mathrm{E}-02$ \\
CVI-PSO & 25000 & 1.724852 & 1.725124 & 1.727665 & $6.120000 \mathrm{E}-04$ \\
\hline \hline
\end{tabular}

Table 11: Results of different algorithms for the welded beam design problem.

\begin{tabular}{l|ccccc}
\hline \hline & FFE & Best & Average & Worst & Std. Dev. \\
\hline SC-PSO-3P & 50000 & 0.0126687 & 0.0128715 & 0.0137703 & $2.538650 \mathrm{E}-04$ \\
CPSO & 200000 & 0.0126747 & 0.0127300 & 0.0129240 & $5.190000 \mathrm{E}-05$ \\
HPSO & 81000 & 0.0126652 & 0.0127072 & 0.0127191 & $1.580000 \mathrm{E}-05$ \\
AATM & 25000 & 0.0126682 & 0.0127080 & 0.0128613 & $4.500000 \mathrm{E}-05$ \\
T-Cell & 36000 & 0.0126650 & 0.0127320 & 0.0133090 & $9.400000 \mathrm{E}-05$ \\
CVI-PSO & 25000 & 0.0126655 & 0.0127310 & 0.0128426 & $5.500000 \mathrm{E}-05$ \\
\hline \hline
\end{tabular}

Table 12: Results of different algorithms for the tension-compression string problem. 


\begin{tabular}{c|rr}
\hline \hline Problem & Low limit & Upper limit \\
\hline 1 & -13.69776169 & -14.62498168 \\
2 & -0.524584754 & -0.599460572 \\
3 & -1.000298967 & -1.00048471 \\
4 & -30665.24483 & -30665.34067 \\
5 & 5174.327258 & 5148.021136 \\
6 & -6959.868837 & -6960.770545 \\
7 & 28.99774975 & 27.28710176 \\
8 & -0.095824409 & -0.095824666 \\
9 & 681.3461332 & 681.0367763 \\
10 & 7618.087189 & 7321.516701 \\
11 & 0.750001544 & 0.7499987 \\
12 & -0.999994195 & -0.999996666 \\
13 & 0.056508561 & 0.055016703 \\
14 & -47.83817353 & -48.08651249 \\
15 & 962.6392106 & 961.9545713 \\
16 & -1.76784791 & -1.903749563 \\
17 & 8955.82206 & 8848.421709 \\
18 & -0.684804785 & -0.777015372 \\
19 & 38.29876149 & 34.66589102 \\
21 & 794.7558171 & 661.3324645 \\
23 & -399.9122071 & -400.0644544 \\
24 & -7 & \\
\hline \hline
\end{tabular}

Table 13: Results of bootstrap test with an $\alpha=0.05$. 


\section{Conclusions and further research}

In this work, a novel fuzzy criterion called System of Convergence SC is proposed, which was implemented using a PSO based algorithm with three phases: 1) Stabilization, 2) Generation and breadth-first search, and 3) Generation and depth-first. This algorithm SC-PSO-3P was tested in a set of benchmark instances for constrained optimization and engineering problems.

As an important remark, a deeper study concerning the boundary conditions of the parameters $a$ and $\alpha$ of SC, as well as their implementation with an algorithm different from PSO-3P must be performed.

The SC-PSO-3P generates best solutions by problems G20 and G22 and G24 that results reported in the literature. Based on information of Table 14, we can say that our method has a behaviour similar that DECV and T-CELL.

\begin{tabular}{l|ccccccc}
\hline \hline & SC-PSO-3P & Memetic & APF-GA & MDE & CVI-PSO & DECV & T-CELL \\
\hline SC-PSO-3P & false & true & true & true & true & false & false \\
Memetic & true & false & false & false & false & true & true \\
APF-GA & true & false & false & false & false & true & true \\
MDE & true & false & false & false & true & true & true \\
CVI-PSO & true & false & false & true & false & false & false \\
DECV & false & true & true & true & false & false & false \\
T-CELL & false & true & true & true & false & false & false \\
\hline \hline
\end{tabular}

Table 14: Results of Wilcoxon Rank test for average normalized values.

It is also necessary to conduct a further study about the topology generated by SC. It was observed that in all the cases studied, SC can reach the global optimum or being very close to it with shorter iteration times and less iterations.

\section{A Proof of theorem 1}

Proof. $G$ is injective by construction.

$G$ is surjective:

Let $g \in \mathbb{R}$, to prove that there is $\tilde{\mathbf{B}}_{g} \subset C(\tilde{\mathbf{B}})$ such that:

$$
G(\tilde{B})=g, \forall \tilde{B} \in \tilde{\mathbf{B}}_{g}
$$

it will be proven by construction. 
Let $\tilde{\mathbf{B}}_{g}=\left\{\tilde{B}=\left(a, f_{1}, f_{2}, f_{3}, \alpha ; w\right)\right\}$ which satisfy the conditions of theorem 1 , and let:

$$
\begin{aligned}
\alpha & =\frac{2 F_{2}}{F_{3}} \\
a & =\frac{4 F_{2}^{2}+A^{-1} F_{3} g}{6 F_{1} F_{3}} .
\end{aligned}
$$

By definition it is known that:

$$
\begin{aligned}
G(\tilde{B}) & =A\left[\alpha^{2} F_{3}-4 \alpha F_{2}+6 a F_{1}\right] \\
& =A\left[\left(\frac{2 F_{2}}{F_{3}}\right)^{2} F_{3}-4 \frac{2 F_{2}}{F_{3}} F_{2}+6 \frac{4 F_{2}^{2}+A^{-1} F_{3} g}{6 F_{1} F_{3}} F_{1}\right] \\
& =A\left[\frac{4 F_{2}^{2}}{F_{3}}-\frac{8 F_{2}^{2}}{F_{3}}+\frac{4 F_{2}^{2}}{F_{3}}+A^{-1} g\right]=g .
\end{aligned}
$$

Therefore, function $G$ is bijective by classes.

\section{B Proof of proposition 1}

Proof. Let $\tilde{B} \in C(\tilde{\mathbf{B}})$ such that $\tilde{B}=\left(a, f_{1}, f_{2}, f_{3}, \alpha ; w\right)$, and $s \in \mathbb{R}$ then:

$$
\tilde{B} s=\left(a s, f_{1}, f_{2}, f_{3}, \alpha s ; w\right) .
$$

Using the definition of $G$ we have that:

$$
\begin{aligned}
G(\tilde{B} s)= & A\left[(\alpha s)^{2} F_{3}-4(\alpha s) F_{2}+6(a s) F_{1}\right] \\
& A\left[\left(\left(\alpha\left(s^{*}+\epsilon\right)\right)^{2} F_{3}-4 \alpha\left(s^{*}+\epsilon\right) F_{2}+6 a\left(s^{*}+\epsilon\right) F_{1}\right]\right. \\
& A\left[\left(\alpha s^{*}\right)^{2} F_{3}+2 \alpha^{2} s^{*} \epsilon F_{3}+(\alpha \epsilon)^{2} F_{3}-4 \alpha s^{*} F_{2}-4 \alpha \epsilon F_{2}\right. \\
& \left.+6 a s^{*} F_{1}+6 a \epsilon F_{1}\right] \\
= & A\left[\left(\alpha s^{*}\right)^{2} F_{3}-4 \alpha s^{*} F_{2}+6 a s^{*} F_{1}\right] \\
& +A\left[2 \alpha^{2} s^{*} \epsilon F_{3}+(\alpha \epsilon)^{2} F_{3}-4 \alpha \epsilon F_{2}+6 a \epsilon F_{1}\right] .
\end{aligned}
$$

Therefore:

$$
\begin{aligned}
\lim _{\epsilon \rightarrow 0} G(\tilde{B} s)= & \lim _{\epsilon \rightarrow 0} A\left[\left(\alpha s^{*}\right)^{2} F_{3}-4 \alpha s^{*} F_{2}+6 a s^{*} F_{1}\right] \\
& +\lim _{\epsilon \rightarrow 0} A\left[2 \alpha^{2} s^{*} \epsilon F_{3}+(\alpha \epsilon)^{2} F_{3}-4 \alpha \epsilon F_{2}+6 a \epsilon F_{1}\right] \\
= & G\left(\tilde{B} s^{*}\right) .
\end{aligned}
$$

Rev.Mate.Teor.Aplic. (ISSN print: 1409-2433; online: 2215-3373) Vol. 23(1): 111-142, January 2016 


\section{Proof of proposition 2}

Proof. It will be demonstrated by mathematical induction.

First it will be proven for $k=2$ :

$$
\begin{aligned}
G\left(\tilde{B}_{1} s_{1}+\tilde{B}_{2} s_{2}\right) \\
=A\left[\left(s_{1} \alpha_{1}+s_{2} \alpha_{2}\right)^{2} F_{3}-4\left(s_{1} \alpha_{1}+s_{2} \alpha_{2}\right) F_{2}+6\left(s_{1} a_{1}+s_{2} a_{2}\right) F_{1}\right] \\
=A\left[\left(\left(s_{1}^{*} \alpha_{1}\right)^{2}+2 s_{1}^{*} \alpha_{1} s_{2}^{*} \alpha_{2}+\left(s_{2}^{*} \alpha_{2}\right)^{2}\right) F_{3}\right. \\
\left.\quad-4\left(s_{1}^{*} \alpha_{1}+s_{2}^{*} \alpha_{2}\right) F_{2}+6\left(s_{1}^{*} a_{1}+s_{2}^{*} a_{2}\right)\right] \\
=A\left[\left(s_{1}^{*} \alpha_{1}+s_{2}^{*} \alpha_{2}\right)^{2} F_{3}-4\left(s_{1}^{*} \alpha_{1}+s_{2}^{*} \alpha_{2}\right) F_{2}\right. \\
\left.\quad+6\left(s_{1}^{*} a_{1}+s_{2}^{*} a_{2}\right) F_{1}\right]+A\left[H\left(\epsilon_{1}, \epsilon_{2}\right)\right]
\end{aligned}
$$

where:

$$
\begin{aligned}
& H\left(\epsilon_{1}, \epsilon_{2}\right) \\
&=\left(2 \epsilon_{1} s_{1}^{*} \alpha_{1}+\left(\epsilon_{1} \alpha_{1}\right)^{2}+2 \alpha_{1} \alpha_{2}\left(s_{1}^{*} \epsilon_{2}+s_{2}^{*} \epsilon_{1}+\epsilon_{1} \epsilon_{2}\right)+2 \epsilon_{2} s_{2}^{*} \alpha_{2}\right. \\
&\left.+\left(\epsilon_{2} \alpha_{2}\right)^{2}\right) F_{3}-4\left(\epsilon_{1} \alpha_{1}+\epsilon_{2} \alpha_{2}\right) F_{2}+6\left(\epsilon_{1} a_{1}+\epsilon_{2} a_{2}\right) .
\end{aligned}
$$

Therefore:

$$
\begin{aligned}
& \lim _{\left\{\epsilon_{1}, \epsilon_{2}\right\} \rightarrow 0} G\left(\tilde{B}_{1} s_{1}+\tilde{B}_{2} s_{2}\right) \\
= & \lim _{\left\{\epsilon_{1}, \epsilon_{2}\right\} \rightarrow 0} A\left[\left(s_{1}^{*} \alpha_{1}+s_{2}^{*} \alpha_{2}\right)^{2} F_{3}-4\left(s_{1}^{*} \alpha_{1}+s_{2}^{*} \alpha_{2}\right) F_{2}\right. \\
& \left.+6\left(s_{1}^{*} a_{1}+s_{2}^{*} a_{2}\right) F_{1}\right]+\lim _{\left\{\epsilon_{1}, \epsilon_{2}\right\} \rightarrow 0} A\left[H\left(\epsilon_{1}, \epsilon_{2}\right)\right] \\
= & G\left(\tilde{B}_{1} s_{1}^{*}+\tilde{B}_{2} s_{2}^{*}\right) .
\end{aligned}
$$

Using mathematical induction, it is assumed true for $k=n-1$ and will be proven for $k=n$.

Note that $\forall i=1,2, \ldots, k \leq n-1$ it is true that:

$$
G\left(\sum_{i=1}^{k}\left(\tilde{B}_{i} s_{i}\right)^{2}\right)=G\left(\sum_{i=1}^{k}\left(\tilde{B}_{i} s_{i}^{*}\right)^{2}\right)+A\left[H\left(\left\{\epsilon_{i}\right\}_{i=1}^{k}\right)\right]
$$

such that

$$
\lim _{\left\{\epsilon_{i}\right\} \rightarrow 0} H\left(\left\{\epsilon_{i}\right\}_{i=1}^{k}\right)=0 .
$$

For $k=n$, we have:

$$
\text { let } \tilde{B}=\sum_{i=1}^{n} \tilde{B}_{i} s_{i}, \text { such that } \tilde{B}_{i} \in C(\tilde{\mathbf{B}}), i=1,2, \ldots, n
$$

It can be seen that: 
1. $\tilde{B}=\sum_{i=1}^{n} \tilde{B}_{i} s_{i}=\sum_{i=1}^{n-1} \tilde{B}_{i} s_{i}+\tilde{B}_{n} s_{n}=\tilde{B}_{n-1}^{\prime}+\tilde{B}_{n} s_{n}$,

2. $\alpha=\sum_{i=1}^{n-1}\left(\alpha_{i} s_{i}\right)+\alpha_{n} s_{n}=\left(\alpha_{n-1}^{\prime}\right)+\alpha_{n} s_{n}$,

3. $a=\sum_{i=1}^{n-1}\left(a_{i} s_{i}\right)+a_{n} s_{n}=\left(a_{n-1}^{\prime}\right)+a_{n} s_{n}$.

Therefore:

$$
\begin{aligned}
G\left(\sum_{i=1}^{n} \tilde{B}_{i} s_{i}\right)= \\
A\left[\left(\alpha_{n-1}^{\prime}+s_{n} \alpha_{n}\right)^{2} F_{3}-4\left(\alpha_{n-1}^{\prime}+s_{n} \alpha_{n}\right) F_{2}+\right. \\
\left.+6\left(a_{n-1}^{\prime}+s_{n} a_{n}\right) F_{1}+H\left(\left\{\epsilon_{i}\right\}_{i=1}^{n-1}\right)\right] \\
=\quad A\left[\left(\alpha_{n-1}^{\prime}+\left(s_{n}^{*}+\epsilon_{n}\right) \alpha_{n}\right)^{2} F_{3}-4\left(\alpha_{n-1}^{\prime}+\left(s_{n}^{*}+\epsilon_{n}\right) \alpha_{n}\right) F_{2}+\right. \\
\left.+6\left(a_{n-1}^{\prime}+s_{n} a_{n}\right) F_{1}+H\left(\left\{\epsilon_{i}\right\}_{i=1}^{n-1}\right)\right] \\
=\quad A\left[\left(\left(\alpha_{n-1}^{\prime}\right)^{2}+\left(s_{n}^{*} \alpha_{n}\right)^{2}+2 \alpha_{n-1}^{\prime} s_{n}^{*} \alpha_{n}\right) F_{3}-4\left(\alpha_{n-1}^{\prime}+s_{n}^{*} \alpha_{n}\right) F_{2}+\right. \\
+6\left(\left(a_{n-1}^{\prime}+s_{n}^{*} a_{n}\right) F_{1}+H\left(\left\{\epsilon_{i}\right\}_{i=1}^{n-1}\right)+H\left(\epsilon_{n}\right)\right] \\
=\quad A\left[\left(\sum_{i=1}^{n}\left(\alpha_{i} s_{i}^{*}\right)^{2}\right) F 3-4\left(\sum_{i=1}^{n}\left(\alpha_{i} s_{i}^{*}\right)\right) F_{2}+\right. \\
\left.+\left(\sum_{i=1}^{n} a_{i} s_{i}^{*}\right) F_{1}+H\left(\left\{\epsilon_{i}\right\}_{i=1}^{n-1}\right)+H\left(\epsilon_{n}\right)\right]
\end{aligned}
$$

where:

$$
H\left(\epsilon_{n}\right)=\epsilon_{n}\left(2 s_{n}^{*} \alpha_{n}^{2}+2 \alpha_{n-1}^{\prime} \alpha_{n}+\alpha_{n}^{2} \epsilon_{n}+\alpha_{n} F_{2}+a_{n} F_{1}\right) .
$$

Hence:

$$
\begin{aligned}
\lim _{\left\{\epsilon_{i}\right\}_{i=1}^{n} \rightarrow 0} G\left(\sum_{i=1}^{n} \tilde{B}_{i} s_{i}\right) \\
=\lim _{\left\{\epsilon_{i}\right\}_{i=1}^{n} \rightarrow 0} A\left[\left(\sum_{i=1}^{n}\left(\alpha_{i} s_{i}^{*}\right)^{2}\right) F 3-4\left(\sum_{i=1}^{n}\left(\alpha_{i} s_{i}^{*}\right)\right) F_{2}+\right. \\
\left.\quad+\left(\sum_{i=1}^{n} a_{i} s_{i}^{*}\right) F_{1}+H\left(\left\{\epsilon_{i}\right\}_{i=1}^{n-1}\right)+H\left(\epsilon_{n}\right)\right] \\
=G\left(\sum_{i=1}^{n} \tilde{B}_{i} s_{i}^{*}\right)
\end{aligned}
$$

Rev.Mate.Teor.Aplic. (ISSN print: 1409-2433; online: 2215-3373) Vol. 23(1): 111-142, January 2016 


\section{Proof of proposition 3}

Proof. The proof will be done by contradiction.

Suppose:

1. $y^{*}=\left(y_{1}^{*}, y_{2}^{*}, \ldots, y_{k}^{*}\right) \in \mathrm{PF}$

2. $y^{*} \notin \mathrm{SC}-\mathrm{F}$

Then we have:

1. $y^{*} \in \operatorname{Image}(h)$, and $y^{*}$ is non dominated.

2. $\exists \epsilon>0$, such that if $\left\|y^{*}-y\right\| \leq \epsilon \Rightarrow y \in \operatorname{Image}(h)$.

consider a point: $y_{0}=\left(y_{1}^{*}-\frac{\epsilon}{\sqrt{k}}, y_{2}^{*}-\frac{\epsilon}{\sqrt{k}}, \ldots, y_{k}^{*}-\frac{\epsilon}{\sqrt{k}}\right)$. Note that:

$$
\left\|y^{*}-y_{0}\right\|=\sqrt{\left(\frac{\epsilon}{\sqrt{k}}\right)^{2}+\ldots+\left(\frac{\epsilon}{\sqrt{k}}\right)^{2}}=\epsilon,
$$

for that reason $y_{0} \in \operatorname{Image}(h)$ and $y_{0}$ dominates to $y^{*}$, which is a contradiction, therefore: $\mathrm{PF} \subseteq \mathrm{SC}-\mathrm{F}$.

\section{Acknowledgements}

The authors wish to thank the three anonymous referees since their comments helped to considerably improve the present work.

S.G. de C. would also like to thank D.Sto. and to P.V.Gpe. for their inspiration, and to Ma, Ser, Mon, Chema, and to his Flaquita for all their support

\section{References}

[1] Aragon, V.S.; Coello, C.A.C. (2010) "A modified version of a t-cell algorithm for constrained optimization problems", Int. J. Numer. Methods Eng. 84(3): 351-378.

[2] Belegundu, A.D. (1982) A Study of Mathematical Programming Methods for Structural Optimization. Ph.D. Thesis, Department of Civil Environmental Engineering, University of Iowa, Iowa City.

[3] Coello, C.A.C. (2000) "Use of a self-adaptive penalty approach for engineering optimization problems", Comput. Ind. 41(2): 113-127. 
[4] Deb, K. (1991) "Optimal design of a welded beam via genetic algorithms", AIAA J. 29(11): 2013-2015.

[5] de-los-Cobos-Silva, S.G. (2015) "SC: system of convergence. Theory and fundaments", Revista de Matemática: Teoría y Aplicaciones 22(2): 341367.

[6] Dubois, D.; Prade, H. (1978) “Operations on fuzzy numbers”, International Journal of Systems Science 9(6): 613-626.

[7] Elsayed, S.M.; Sarker, R.A.; Essam, D.L. (2012) "On an evolutionary approach for constrained optimization problem solving", Applied Soft Computing 12(10): 3208-3227.

[8] Gavana, A. (2007?) "Global optimization benchmarks and AMPGO. Test functions", in: http://infinity77.net/global_ optimization/

[9] Glover, F. (1989) “Tabu search, Part I”, ORSA Journal on Computing 1(3): 190-206.

[10] Glover, F. (1998) "A template for scatter search and path relink", in: J.K. Hao, E. Lutton, E. Ronald, M. Schoenauer \& D. Snyers (Eds.) Artificial Evolution, Lecture Notes on Computer Science 1363, Springer, Berlin: 151.

[11] He, Q.; Wang, L. (2007a) "An effective co-evolutionary particle swarm optimization for constrained engineering design problems", Eng. Appl. Artif. Intell. 20(1): 89-99.

[12] He, Q.; Wang, L. (2007b) "A hybrid particle swarm optimization with a feasibility-based rule for constrained optimization", Appl. Math. Comput. 186(2): 1407-1422.

[13] Hedar, A.R. (2007?) "Global optimization test problems", in: http://www-optima.amp.i.kyoto-u.ac.jp/member/ student/hedar/Hedar_files/go.htm

[14] Hibbeler, R.C. (2000) Mechanics of Materials, 8th ed. Prentice Hall, New Jersey.

[15] Holland, J.H. (1975) Adaptation in Natural and Artificial Systems. University of Michigan Press, Ann Arbor MI. 
[16] Imran, M.; Hashima, R.; Khalid, N.E.A. (2013) "An overview of particle swarm optimization variants", Procedia Engineering 53: 491-496.

[17] Kalami Heris, S.M. (s.f.) “S. Mostapha Kalami Heris' homepage”, http: //www.kalami.ir

[18] Kannan, B.K.; Kramer, S.N. (1994) "An augmented Lagrange multiplier based method for mixed integer discrete continuous optimization and its applications to mechanical design", J. Mech. Des. 116(2): 405-411.

[19] Kennedy, J.; Eberhart, R. (1995) "Particle swarm optimization", in: Proc. of the IEEE International Conference on Neural Networks, vol. 4: 19421948.

[20] Kennedy, J.; Eberhart, R.C.; Shi, Y. (2001) Swarm Intelligence. Morgan Kaufmann, San Francisco.

[21] Kirkpatrick, S.; Gelatt, C.; Vecchi, M. (1983) "Optimization by simulated annealing", Science 220: 671-680.

[22] Liang, J.J.; Runarsson, T.P.; Mezura-Montes, E.; Clerc, M.; Suganthan, P.N.; Coello Coello, C.A.; Deb, K. (2006) "Problem definitions and evaluation criteria for the CEC 2006, special session on constrained realparameter optimization", Technical Report, IEEE Congress on Evolutionary Computation, 24 pp.

[23] Liou, T.S.; Wang, M.J.J. (1992) "Ranking fuzzy numbers with integral value", Fuzzy Sets and Systems 50(3): 247-255.

[24] Liu, C.A. (2007) "New multiobjective PSO algorithm for nonlinear constrained programming problems", in: R. Wang, E. Shen E. \& F. Gu (Eds.) Advances in Cognitive Neurodynamics ICCN: 955-962.

[25] Liu, H.; Cai, Z.; Wang, Y. (2010) "Hybridizing particle swarm optimization with differential evolution for constrained numerical and engineering optimization", Appl. Soft Comput. 10(2): 629-640.

[26] Lu, H.; Chen, W. (2008) "Self-adaptive velocity particle swarm optimization for solving constrained optimization problems", J. Glob. Opt. 41(3): $427-445$.

[27] Mazhoud, I.; Hadj-Hamou, K.; Bigeon J.; Joyeux P. (2013) “Particle swarm optimization for solving engineering problems: a new constraint-handling mechanism", Eng. Appl. of Art. Intel. 26(4): 1263-1273. 
[28] Mezura-Montes, E.; Velázquez-Reyes, J.V.; Coello-Coello, C.A. (2006) "Modified differential evolution for constrained optimization", in: IEEE Congress on Evolutionary Computation: 25-32.

[29] Mezura-Montes, E.; Miranda-Varela, M.E.; Gómez-Ramón, R.C. (2010) "Differential evolution in constrained numerical optimization: An empirical study", Inf. Sci. 180(22): 4223-4262.

[30] Rao, S.S. (1996) Engineering Optimization: Theory and Practice. Wiley, New York.

[31] Sedighizadeh, D.; Masehian, E. (2009) "Particle swarm optimization methods, taxonomy and applications", Int. Journal of Computer Theory and Engineering 1(5): 486-502.

[32] Tessema, B.; Yen, G.G. (2009) "An adaptive penalty formulation for constrained evolutionary optimization”, IEEE Transactions on Systems, Man and Cybernetics, Part A: Systems and Humans 39(3): 565-578.

[33] Toscano-Pulido, G.; Coello, C.A.C. (2004) "A constraint-handling mechanism for particle swarm optimization”, in: IEEE Congress on Evolutionary Computation, vol. 2, IEEE Press: 1396-1403.

[34] Zitzler, E.; Deb, K.; Thiele, L. (2000) "Comparison of multiobjective evolutionary algorithm: empirical results”, Evolutionary Computation 8(2): 173-195

[35] Wang, Y.; Cai, Z.; Zhou, Y. (2009) "Accelerating adaptive trade-off model using shrinking space technique for constrained evolutionary optimization”, Int. J. Numer. Methods Eng. 77(11): 1501-1534. 